\title{
L'enseignement des langues régionales en France aujourd'hui : état des lieux et perspectives
}

\section{Carmen Alen-Garabato et Micheline Cellier}

\section{OpenEdition}

\section{Journals}

Édition électronique

URL : http://journals.openedition.org/trema/903

DOI : 10.4000/trema.903

ISSN : 2107-0997

Éditeur

Faculté d'Éducation de l'université de Montpellier

Édition imprimée

Date de publication : 1 septembre 2009

Pagination : 1 - 4

ISSN : 1167-315X

\section{Référence électronique}

Carmen Alen-Garabato et Micheline Cellier, «L'enseignement des langues régionales en France aujourd'hui : état des lieux et perspectives », Tréma [En ligne], 31 | 2009, mis en ligne le 20 septembre 2010, consulté le 22 septembre 2020. URL : http://journals.openedition.org/trema/903 ; DOI : https:// doi.org/10.4000/trema.903

Ce document a été généré automatiquement le 22 septembre 2020.

Trema 


\title{
L'enseignement des langues régionales en France aujourd'hui : état des lieux et perspectives
}

\author{
Carmen Alen-Garabato et Micheline Cellier
}

1 L'histoire de l'enseignement des langues régionales à l'école publique (ou privée) en France n'a qu'une cinquantaine d'années et s'est construite petit à petit grâce aux efforts constants des militants et des amoureux de ces langues, à contre courant de l'idéologie unilinguiste qui habite les décideurs mais aussi la société civile française en général. On sait qu’à partir de la Révolution française, une politique linguistique ayant pour objectif non seulement « d'universaliser l'usage de la langue française» mais aussi « d'anéantir les patois » est annoncée et

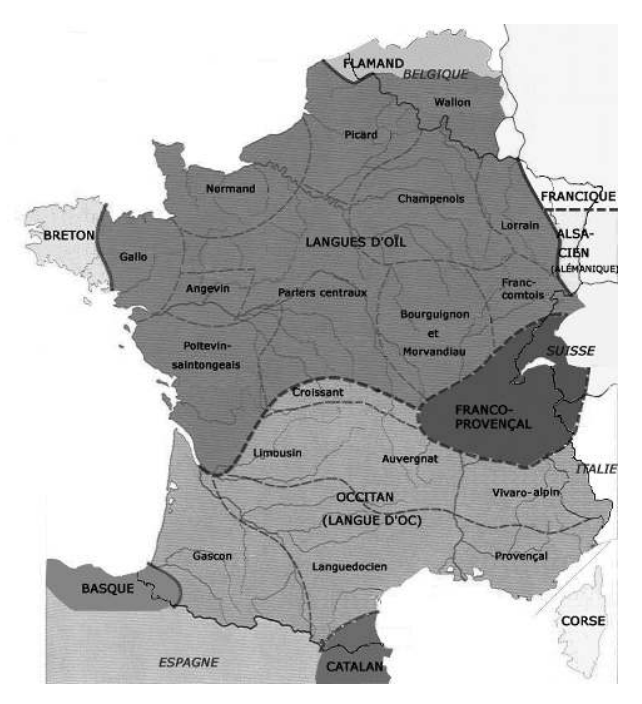
commence à être appliquée. Peu à peu, un monolinguisme presque complet en français s'est installé dans le territoire national. La Loi FERRY de scolarisation obligatoire, laïque et gratuite (1881) a fait de l'école le principal acteur de la francisation des enfants (surtout dans les campagnes): l'apprentissage efficace du français y a été accompagné d'une dévalorisation et d'une stigmatisation (aussi efficaces) des langues régionales.

2 Enseigner les langues régionales aujourd'hui signifie aller à l'encontre d'une idéologie vieille de plus de deux siècles et affronter représentations et stéréotypes négatifs. Cette idéologie freine toute tentative (sincère ou non) de donner une vraie existence juridique aux langues régionales en France. Les propos du fameux Rapport de l'abbé Grégoire (1791) resurgissent chaque fois que le débat sur les langues régionales se 
rouvre (celui provoqué par la signature de la Charte européenne des langues régionales en est un bon exemple $\left.{ }^{1}\right)$ : les langues régionales sont des restes du passé, du folklore, de l'ignorance ... l'affaire des militants suspects d'anti - républicanisme face au français qui est la langue de la modernité, de la science, de l'ouverture, la langue de la République, comme l'affirme par ailleurs la Constitution en son article 2 depuis 1992.

Le besoin d'affirmer une identité plurilingue face à la mondialisation et de renforcer la crédibilité internationale de la France dans sa défense de la francophonie ${ }^{2}$ a sûrement fait avancer quelque peu la question des langues régionales dans l'Hexagone mais n'a pas évincé jusqu'à aujourd'hui le poids de l'idéologie unilinguiste.

Dans ce contexte, on peut bien comprendre toute la difficulté de l'enseignement des langues régionales et encore plus de l'enseignement en langues régionales en France: un vrai parcours du combattant.

5 La loi DEIXONNE (Loi n $51-48$ du 11 janvier 1951 relative à l'enseignement des langues et dialectes locaux) est la première, et la seule jusqu'à aujourd'hui, spécifique à l'enseignement des langues régionales. Elle constitue une reconnaissance officielle de l'existence de certaines langues régionales (l'occitan, le breton, le basque et le catalan ; d'autres langues seront concernées plus tard), jusque - là ignorées dans les textes officiels.

6 Un long chemin a été parcouru depuis cette loi $^{3}$. D'après les chiffres livrés par la DGLFLF en 20074, durant l'année scolaire 2005 - 2006 : 404351 élèves ont bénéficié d'un enseignement en / de langue régionale (toutes formes d'enseignement confondues, public et privé sous contrat) : 282894 à l'école, 96295 au collège et 25162 en lycée. La liste des langues concernées s'est aussi élargie : basque, breton, catalan, corse, créole, gallo, occitan / langue d'oc, langues régionales d'Alsace, langues régionales des pays mosellans, tahitien et langues mélanésiennes.

7 L'année 2008 a mis les langues de France au centre du débat public, d'abord à l'Assemblée nationale (le 7 mai), puis au Sénat (le 13 mai). Même si la ratification de la Charte européenne des langues régionales ou minoritaires n'est toujours pas envisagée, un projet de loi relatif à l'enseignement, aux médias, à la culture et aux services publics devrait être présenté aux Assemblées en 2009. Mais déjà, depuis juillet 2008, les langues régionales sont inscrites dans la Constitution : non pas dans l'article 1er (amendement adopté par les députés le 22 mai et rejeté par le Sénat le 18 juin), mais dans le titre XII relatif aux collectivités territoriales, article $75-1$ (d'après le texte adopté par le congrès le 21 juillet): «Les langues régionales appartiennent au patrimoine de la France ».

Dans la conjoncture actuelle, il a paru intéressant ${ }^{5}$ de faire, dans ce numéro de Tréma, l'état des lieux de l'enseignement des langues régionales en 2009.

Avant l'analyse consacrée à chacune d'entre elles, les deux premiers articles engagent le propos de façon générale, avec des perspectives différentes. 
10 L'une, d'ordre historique, permet à James COSTA de faire le point, de manière analytique, sur un siècle de travaux consacrés aux langues régionales dans le cadre de l'INRP, travaux qui reflètent la place qu'elles occupent dans le champ didactique - elles sont fortement rattachées à la réflexion sur la variation langagière, depuis les années 80 - et dans la représentation nationale. Leur enseignement n'est pas encore totalement légitimé et devrait, pour garder sa vitalité, s'intégrer à celui, plus large, des autres disciplines linguistiques.

11 Le deuxième angle d'attaque est d'ordre bibliographique. L'article de Pierre BOUTAN et Micheline CELLIER rend compte de la nécessité de recenser, dans une base de données informatisée et accessible par Internet, les outils pédagogiques qui permettent d'enseigner les langues régionales - parlées dans l'Hexagone et en Corse -, leurs cultures et leurs territoires, avec deux publics cibles : d'une part, les enseignants qui profitent d'un large corpus d'ouvrages référencés et analysés; d'autre part, les chercheurs qui pourront initier des études comparatives entre les différentes didactiques.

Les neuf autres articles déclinent les situations spécifiques à chaque domaine linguistique. L'ordre retenu pour la présentation des langues régionales correspond à celui de leur entrée dans l'enseignement, d'abord celles qui sont citées dans la loi DEIXONNE de 1951 puis celles qui les ont rejointes par décret, au fur et à mesure. Le gallo constitue un cas particulier car il est exclu de la liste des langues retenues par l'arrêté ministériel de 2005 mais il est en option au baccalauréat.

Il a été rapproché du breton car les deux sont étiquetés « langues de Bretagne ».

Stefan MOAL - MORROW pour le breton, André LE COQ pour le gallo, Jakes SARRAILLET pour le basque, Mary SANCHIZ et Luc BONET pour le catalan, Marie - Jeanne VERNY pour l'occitan, Alain DI MEGLIO pour le corse, Louise PELTZER pour le tahitien et les langues polynésiennes, Daniel MORGEN et Armand ZIMMER pour les langues régionales d'Alsace et des pays mosellans, Evelyne ADELIN et Mylène LEBON - EYQUEM pour le créole vont tour à tour dresser un bilan de l'enseignement de la langue dont ils sont spécialistes et envisager des perspectives pour son développement.

Leurs interventions se construisent autour de quelques axes communs.

Certains relèvent de l'aspect organisationnel et administratif.

Il s'agit de faire le point sur l'état de l'enseignement, en termes quantitatifs d'abord, en déclinant le nombre d'élèves et d'enseignants dans les différents niveaux, l'évolution des effectifs dans les dernières années et les rapports entre l'offre et la demande. La description porte également sur la répartition entre les secteurs privé, public et associatif sous contrat et les modalités d'enseignement proposées - option, cursus bilingue, immersion - ainsi que d'une manière plus large, sur le niveau d'engagement des collectivités locales dans le financement, et les éventuelles conventions spécifiques entre l'Etat et les collectivités territoriales ou les régions d'un pays voisin.

D'autres points permettent d'aborder l'aspect plus pédagogique et didactique: les difficultés spécifiques à chaque domaine linguistique en ce qui concerne les cursus, les examens, la formation initiale et continue. L'aspect sociolinguistique transparaît à travers les questions des représentations, de la présence de la langue et de la norme. 


\section{NOTES}

1. Les mots de Stefan OETER, membre du Comité d'experts indépendants de la Charte, professeur de droit international à l'Université de Hambourg, résument bien le débat: "En lisant les différentes opinions caractéristiques de la discussion sur l'impact de la Charte en France, on pourrait avoir l'impression que la Charte constitue un instrument de révolution. La Charte apparaît, selon l'opinion d'une tendance importante, comme étant le cheval de Troie désigné pour détruire les éléments essentiels de la Constitution républicaine. La République une et indivisible paraît menacée » (S. OETER (2002), «L'impact de la mise en œuvre de la Charte », La Charte européenne des langues régionales ou minoritaires et la France. Quelle(s) langue(s) pour la République ? Dilemme "Diversité / unicité ». Colloque organisé par le Conseil de l'Europe et l'Université Robert - Schuman de Strasbourg, Strasbourg, 11 - 12 avril 2002, Strasbourg : Éditions du conseil de l'Europe : 75 - 83).

2. Cf. la conclusion du Rapport Poignant: «Le choix de la francophonie : la langue française a besoin d'être défendue et développée à l'étranger. Elle est notre langue commune. Son rayonnement à l'extérieur comme langue étrangère, sa défense comme langue minoritaire seront d'autant plus convaincants que la place des langues de France sera affirmée. Nous sommes un pays qui connaît une grande diversité de langues parlées, qu'elles soient historiques ou issues de mouvements migratoires. C'est pour cela que la langue française est primordiale, c'est pour cela qu'il faut avoir une politique suivie et cohérente pour les autres ".

3. Pour le répertoire des textes de loi concernant les langues régionales, voir Violaine EYSERIC, Le corpus juridique des langues de France, Délégation générale à la langue française et aux langues de France, 2005.

4. Rapport au Parlement sur l'emploi de la langue française, Délégation générale à la langue française et aux langues de France 2007.

5. À partir d'une idée de Pierre BOUTAN qui a initié dans le groupe de recherche Dipralang des travaux fructueux sur "Enseigner la région» (1999 - 2002 - IUFM de Montpellier - Didaxis Dipralang) et qui a trouvé utile de faire le point actuel sur la question.

\section{AUTEURS}

\section{CARMEN ALEN-GARABATO}

MCF - HDR, Arser - Dipralang, Université Montpellier III

\section{MICHELINE CELLIER}

MCF, IUFM de Montpellier - Université Montpellier 2, Didaxis - DIpralang 